\title{
Ausschreibung der höheren Fachprüfung 2001 zum/zur eidg. dipl. Pharmaberater/in
}

Ort:

Daten:

Vorbereitungskurs:
FEUSI-Bildungszentrum, Hauptgasse 25, 4600 Olten

Schriftliche Prüfung: Samstag, 16. und 23. Juni 2001

Mündliche Prüfung: Donnerstag, 28. Juni 2001

Start 2001

Ort: FEUSI-Bildungszentrum, Hauptgasse 25, 4600 Olten

Bedingungen für die Zulassung zur höheren Fachprüfung: mindestens drei Jahre Erfahrung (bei paramedizinischer Ausbildung zwei Jahre) im Pharma-Aussendienst.

Für weitere Auskünfte und Anforderung des Prüfungsreglementes wenden Sie sich bitte an: Meggy Jäger, Präsidentin der Ethischen Berufsbildungskommission, Brunnenrain 191, 5735 Pfeffikon/LU, Natel 07928539 02, Fax 06277146 82, E-mail: jaeger.cephalon@dplanet.ch

\section{Hilfskasse für Schweizer Ärzte}

\section{Eingegangene Spenden}

Vom 1. Juli bis 30. September 2000 sind 106 Spenden im Gesamtbetrag von Fr. 26 545.- eingegangen. Der Stiftungsrat der Hilfskasse für Schweizer Ärzte freut sich sehr, diese Gaben bekanntgeben zu dürfen, und dankt allen Spenderinnen und Spendern recht herzlich. Damit die Spenden in voller Höhe den Destinatären zukommen, haben wir uns entschlossen, für Spenden unter Fr. 500.- auf den Versand von persönlichen Dankschreiben zu verzichten. Wir hoffen sehr, dass diese Massnahmen bei Ihnen auf Verständnis stösst.

Der Kassier des Stiftungsrates:

Dr. U. Straumann

\section{Caisse de secours des médecins suisses}

\section{Contributions reçues}

Du $1^{\text {er }}$ juillet au 30 septembre 2000, nous avons reçu 106 dons d'une valeur totale de Fr. 26 545.-. Le Conseil de fondation de la Caisse de secours des médecins suisses est très content de pouvoir publier ces chiffres et tient à remercier vivement tous les donateurs. Afin que les contributions puissent être versées entièrement aux destinataires, nous avons pris la décision de renoncer à l'envoi de lettres de remerciement pour les dons inférieurs à Fr. 500.-. Nous espérons que les donateurs feront preuve de compréhension et nous leur adressons encore une fois nos remerciements. 